\title{
Metodologias para monitoramento nutricional de gérbera cultivada em substrato ${ }^{(1)}$
}

\author{
FERNANDA LUDWIG(2); DIRCEU MAXIMINO FERNANDES(3); \\ AMARALINA CELOTO GUERRERO(4); ROBERTO LYRA VILLAS BÔAS ${ }^{(3)}$
}

\begin{abstract}
RESUMO
Este trabalho foi conduzido com o objetivo de avaliar metodologias para o monitoramento nutricional de gérbera cultivada em substrato. O experimento foi conduzido em casa de vegetação, em Botucatu, SP. Foram utilizados cinco formulações de substratos e duas cultivares de gérbera, Red e Cherry, transplantadas em vaso com capacidade de 1 litro. A determinação da condutividade elétrica (CE) e do pH da solução do substrato foi realizada aos 1, 15, 29, 43 e 50 dias após aclimatação (DAA), com o uso das metodologias do "pourthru", diluição de 1:2 (volume de substrato/volume de água) e diluição de 1:5 (massa de substrato/volume de água). Para os métodos de diluição, o substrato foi retirado dos vasos, separado das raízes, homogeneizado e seco ao ar, anteriormente à análise. Em cada avaliação foram utilizados 40 vasos. As correlações lineares estabelecidas entre as metodologias foram obtidas pelo método de Pearson. Os valores de CE da solução do substrato foram superiores quando utilizado o método "pourthru", devido a menor diluição da amostra e natureza de deslocamento da solução no recipiente. Os valores de pH foram semelhantes nos três métodos, os quais apresentaram correlação linear positiva e altamente significativa. Independente do método utilizado, as diferenciações quanto ao $\mathrm{pH}$ dos diferentes substratos foram detectadas ao longo do ciclo. As correlações significativas para os valores de CE e pH validam os métodos. A fim de possibilitar o monitoramento periódico, a análise nutricional e eventual correção pode ser efetuada de maneira mais efetiva pelo método "pourthru", por ser não destrutivo e agilizar a obtenção da resposta.
\end{abstract}

Palavras-chave: Gerbera jamesonii, "pourthru”, extratores aquosos, condutividade elétrica, pH.

\section{ABSTRACT}

Methodologies for nutritional monitoring of gerbera cultivated on substrate

This study aimed to assess methodologies for the nutritional monitoring of gerbera cultivated on substrate. The experiment was carried out in greenhouse, in Botucatu (22 $51^{\prime} \mathrm{S}$ and $48^{\circ} 26^{\prime} \mathrm{W}$ ), São Paulo State, Brazil. Five substrate formulations were used and two gerbera cultivars, Red and Cherry, were transplanted to 1-liter pots. Electrical conductivity (EC) and pH of the substrate solution were determined at 1, 15, 29, 43 and 50 days after acclimation (DAA) by adopting the methodologies "pourthru", dilution of 1:2 (substrate volume/water volume) and dilution of 1:5 (substrate mass /water volume). For the dilution methods, the substrate was removed from the pots, separated from the roots, homogenized and air dried before analysis. In each assessment, 40 pots were used. Linear correlations established among the methodologies were obtained according to the Pearson's method. EC values of the substrate solution were higher when the method "pourthru" was used due to the lower sample dilution and the nature of the solution displacement in the container. Significant correlations validate the methods while for periodic monitoring, nutritional analysis and eventual correction can be more effectively done by adopting the method "pourthru" since the displacement of the substrate solution using the non-destructive method reduces the costs and accelerates the response.

Keywords: Gerbera jamesonii, "pourthru", aqueous extractors, electrical conductivity, $\mathrm{pH}$.

\section{INTRODUÇÃO}

A gérbera é uma planta herbácea, com inflorescência em capítulo e pertencente à família Asteraceae (MERCURIO, 2002). Sua comercialização como flor envasada é recente, e com isso, há uma crescente demanda por substratos adequados ao seu cultivo.

$\mathrm{O}$ uso de substratos em plantas envasadas e cultivadas em casa de vegetação, permite que o produtor possa manter os níveis nutricionais ajustados a cada cultura, através do manejo do $\mathrm{pH}$ e da condutividade elétrica (CAVINS, 2002). O monitoramento regular da condutividade elétrica pode eliminar os problemas associados com a fertilização, pela indicação indireta da disponibilidade de nutrientes para as culturas, pois muitas vezes as análises completas de tecido e substratos tornam-se demoradas e onerosas.

De acordo com Santos (2005) ainda existem poucas informações e muitas controvérsias quanto aos métodos analíticos de avaliação da condutividade elétrica e $\mathrm{pH}$, faltando padronização dos procedimentos que tornem possível a comparação dos resultados e a definição das faixas críticas para sua interpretação e recomendação de correção e adubação.

\footnotetext{
(1) Trabalho recebido para publicação em 11/10/2012 e aprovado em 26/02/2015

(2) Universidade Estadual do Rio Grande do Sul (UERGS) / Unidade em Santa Cruz do Sul. Avenida Independência, 2824. CEP: 96816-501, Santa Cruz do Sul, RS, Brasil. email: fernanda-ludwig@uergs.edu.br

(3) Departamento de Recursos Naturais / Ciência do Solo, FCA/UNESP. Fazenda Experimental Lageado. Caixa Postal 237. CEP: 18610-307, Botucatu, SP, Brasil.

(4) Centro de Ciências e Tecnologia Alimentar / Universidade Federal de Campina Grande. Campus Pombal. Rua Feitosa, 1770. CEP: 58840000, Pombal, PB, Brasil.
} 
Dentre os métodos passíveis de aplicação para esse monitoramento, cita-se o "pourthru", descrito por Cavins (2002) como sendo um método rápido, prático e não destrutivo, que se baseia no deslocamento de parte da solução do substrato, a qual é lixiviada e coletada para análise. De acordo com Cavins et al. (2005) essa extração simples pode ser facilmente realizada na casa de vegetação, sem equipamentos especiais, auxiliando na economia de tempo e permitindo um ajuste imediato da fertilidade. Entretanto, Cavins et al. (2008) citam que a maior limitação é a falta de recomendação de valores padrões para a floricultura, baseados no "pourthru".

Outros métodos baseiam-se na diluição do substrato em extratores aquosos. A diluição de 1:2 (volume/volume) é rotineiramente usada pelas empresas de substrato no Brasil, para determinação de $\mathrm{pH}$ e condutividade elétrica (ABREU et al., 2007) e por produtores de flores e plantas ornamentais. Contudo, esse método geralmente não fornece uma amostra satisfatória para a completa análise nutricional, devido à natureza da diluição (CAVINS et al., 2004). A diluição do 1:5 (massa/volume) foi adotada como padrão para análise de condutividade elétrica e $\mathrm{pH}$ da solução do substrato, aprovado em instituição normativa n.17 de 21 de maio de 2007, pelo Ministério da Agricultura, Pecuária e Abastecimento (BRASIL, 2007) e, paulatinamente, vem sendo adotado pelas empresas de substratos, a fim de padronizar as medidas de caracterização química inicial.

Os métodos de diluição do 1:2 e 1:5 foram considerados eficientes por Abreu et al. (2007), para determinação da condutividade elétrica, $\mathrm{pH}$ e macronutrientes de diversos componentes e substratos. São metodologias conhecidas e apresentam um protocolo mais desenvolvido, porém são destrutivos e por isso sua aplicação se restringe a uma menor área amostral, a fim de reduzir os custos de análise, quando utilizados para monitoramento nutricional de plantas cultivadas em ambiente protegido. De acordo com Mota et al. (2011), por apresentarem metodologias destrutivas, muitas vezes torna-se difícil e onerosa a rotina de análise, além da possibilidade de solubilização de alguns sais, devido ao excesso de diluição. Dessa forma, a utilização dessas metodologias teria maior aplicabilidade para caracterização inicial dos materiais utilizados como substrato, e não para análises de rotina.

São necessárias avaliações que correlacionem estas metodologias, para facilitar a aplicabilidades das mesmas. Desse modo, este trabalho foi conduzido com o objetivo de avaliar metodologias para monitoramento nutricional de gérbera conduzida em substrato.

\section{MATERIAL E MÉTODOS}

O experimento foi conduzido em casa de vegetação com cobertura em arco com plástico transparente de 150 $\mu \mathrm{m}$ de espessura, com laterais de tela branca, cortinas de plástico transparente e pavimentada com concreto, em uma área total de $168 \mathrm{~m}^{2}$ ( 7 x $\left.24 \mathrm{~m}\right)$ e 2,6 m de pé direito, em Botucatu $\left(22^{\circ} 51^{\prime}\right.$ 'S e $\left.48^{\circ} 26^{\prime} \mathrm{W}\right)$, SP.

Segundo a classificação de Koeppen, o clima predominante na região é do tipo Cwa, caracterizado pelo clima tropical de altitude, com inverno seco e verão quente e chuvoso. A temperatura e umidade relativa do ar máxima, média e mínima, no interior da casa de vegetação são apresentadas na Figura 1.
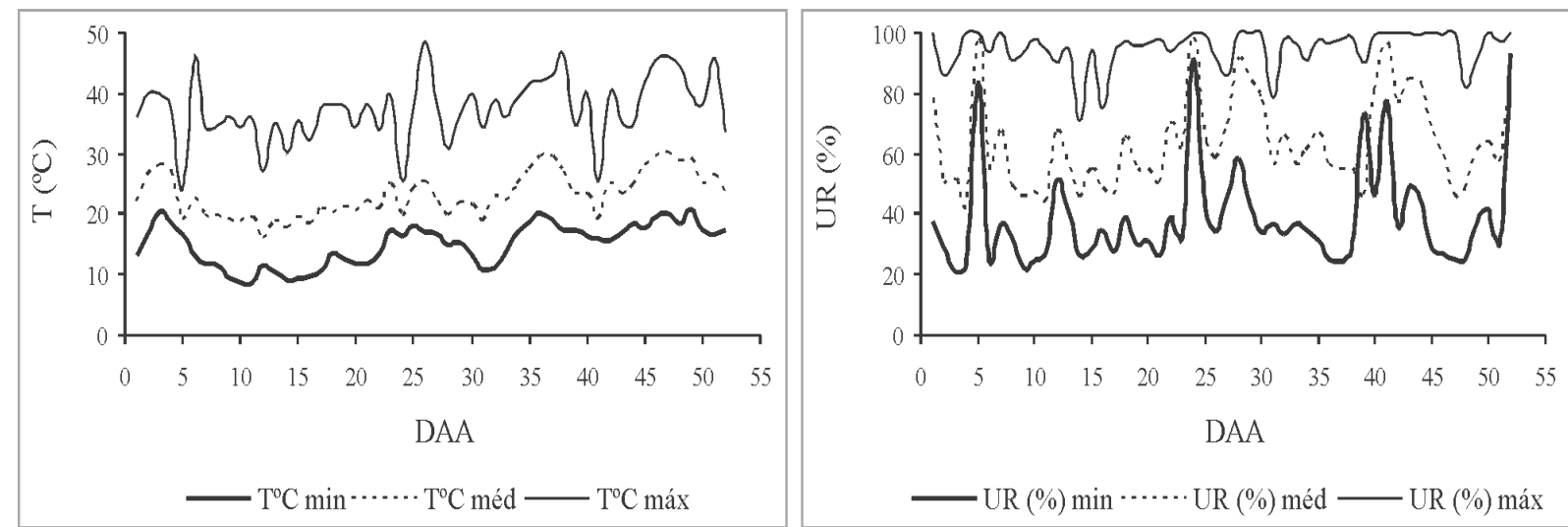

Figura 1. Temperatura $\left({ }^{\circ} \mathrm{C}\right)$ e umidade relativa do ar $(\%)$ mínima, média e máxima ao longo do experimento. Botucatu, SP. (DAA: dias após aclimatação).

Figure 1. Temperature $\left({ }^{\circ} \mathrm{C}\right)$ and relative humidity (\%) minimum, average and maximum along the experiment. Botucatu, SP. (DAA: days after acclimation). 
Os substratos utilizados foram: $1-40 \%$ terra de subsolo (Latossolo Vermelho Escuro), 40\% casca de pinus, 10\% composição $1\left(\mathrm{C}_{1}\right)(40 \%$ casca de pinus $+30 \%$ vermiculita $+30 \%$ casca de arroz carbonizada), $10 \%$ composição $2\left(\mathrm{C}_{2}\right)$ ( $75 \%$ casca de pinus $+25 \%$ acícula de pinus) $; 2$ - 20\% terra de subsolo, $30 \%$ casca pinus, $30 \% \mathrm{C}_{1}, 20 \% \mathrm{C}_{2} ; 3-50 \%$ casca de pinus, $20 \%$ fibra de coco granulada, $30 \% \mathrm{C}_{1} ; 4$ $30 \%$ casca de pinus, $30 \% \mathrm{C}_{1}, 20 \%$ fibra de coco granulada,
$20 \%$ fibra de coco mista; 5 - substrato comercial $(70 \%$ casca de pinus $+15 \%$ turfa $+15 \%$ vermiculita).

As características químicas de CE1:5 e pH1:5 (BRASIL, 2007), macronutrientes $1: 1,5$ e micronutrientes $1: 1,5$ (SONNEVELD e ELDEREN, 1994) e físicas de densidade (BRASIL, 2007) e retenção de água (De BOODT e VERDONCK, 1972), relação C/N e matéria orgânica (RAIJ et al., 2001) dos substratos são apresentadas na Tabela 1.

Tabela 1. Características químicas e físicas dos substratos utilizados no experimento. Botucatu (SP).

Table 1. Chemical and physical characteristics of substrates used in the experiment. Botucatu (SP).

\begin{tabular}{|c|c|c|c|c|c|}
\hline Características & Substrato 1 & Substrato 2 & Substrato 3 & Substrato 4 & Substrato 5 \\
\hline $\mathrm{CE}_{1: 5}\left(\mathrm{dS} \mathrm{m}^{-1}\right)$ & 0,54 & 0,49 & 0,26 & 0,28 & 0,48 \\
\hline $\mathrm{pH}_{1: 5 \text { (inicial) }}$ & 5,13 & 4,81 & 5,74 & 5,74 & 7,58 \\
\hline $\mathrm{pH}_{1: 5 \text { (final) }}$ & 5,42 & 4,86 & 5,63 & 5,59 & 6,89 \\
\hline MO (\%) & 19,00 & 28,00 & 38,00 & 33,00 & 34,00 \\
\hline $\mathrm{C} / \mathrm{N}$ & 10,55 & 15,55 & 21,11 & 18,33 & 18,88 \\
\hline $\mathrm{K}_{1: 1,5}\left(\mathrm{mg} \mathrm{L}^{-1}\right)$ & 70,25 & 86,00 & 45,43 & 56,70 & 176,25 \\
\hline $\mathrm{Ca}_{1: 1,5}\left(\mathrm{mg} \mathrm{L}^{-1}\right)$ & 52,40 & 84,98 & 3,57 & 22,83 & 21,63 \\
\hline $\operatorname{Mg}_{1: 1,5}\left(\mathrm{mg} \mathrm{L}^{-1}\right)$ & 50,15 & 57,60 & 12,13 & 24,45 & 12,83 \\
\hline $\mathrm{Cu}_{1: 1,5}\left(\mathrm{mg} \mathrm{L}^{-1}\right)$ & 0,01 & 0,02 & 0,06 & 0,03 & 0,06 \\
\hline $\mathrm{Fe}_{1: 1,5}\left(\mathrm{mg} \mathrm{L}^{-1}\right)$ & 0,38 & 0,62 & 5,57 & 1,76 & 1,83 \\
\hline $\mathrm{Mn}_{1: 1,5}\left(\mathrm{mg} \mathrm{L}^{-1}\right)$ & 0,19 & 2,48 & 0,20 & 0,38 & 0,84 \\
\hline $\mathrm{Zn}_{1: 1,5}\left(\mathrm{mg} \mathrm{L}^{-1}\right)$ & 0,04 & 0,11 & 0,06 & 0,06 & 0,08 \\
\hline Porosidade total- PT (\%) & 78,79 & 80,62 & 84,28 & 89,18 & 83,51 \\
\hline Sólidos (\%) & 21,21 & 19,38 & 15,72 & 10,82 & 16,49 \\
\hline Espaço de aeração - EA (\%) & 23,50 & 19,33 & 12,76 & 17,15 & 21,10 \\
\hline Água disponível - AD (\%) & 28,74 & 29,40 & 37,34 & 35,80 & 32,90 \\
\hline Água remanescente - AR (\%) & 26,48 & 31,89 & 34,62 & 36,23 & 29,50 \\
\hline Densidade úmida $\left(\mathrm{kg} \mathrm{m}^{-3}\right)$ & 750,00 & 850,00 & 690,00 & 590,00 & 530,00 \\
\hline Densidade seca $\left(\mathrm{kg} \mathrm{m}^{-3}\right)$ & 538,00 & 540,00 & 450,00 & 298,00 & 340,00 \\
\hline
\end{tabular}

Substratos: 1: 40\% terra de subsolo (Latossolo Vermelho Escuro (LVE)), 40\% casca de pinus (CP), 10\% composição 1 $\left(\mathrm{C}_{1}\right)(40 \% \mathrm{CP}+30 \%$ vermiculita $(\mathrm{V})+30 \%$ casca de arroz carbonizada $), 10 \%$ composição $2\left(\mathrm{C}_{2}\right)(75 \% \mathrm{CP}+25 \%$ acícula de pinus); 2: 20\% LVE, 30\% CP, 30\% $\mathrm{C}_{1}, 20 \% \mathrm{C}_{2} ; 3: 50 \% \mathrm{CP}, 20 \%$ fibra de coco granulada (FCG), 30\% $\mathrm{C}_{1} ; 4: 30 \%$ $\mathrm{CP}, 30 \% \mathrm{C}_{1}, 20 \% \mathrm{FCG}, 20 \%$ fibra de coco mista; 5: substrato comercial (70\% CP $+15 \%$ turfa $\left.+15 \% \mathrm{~V}\right)$. CE: eletrical condutivity, PT: total porosity, S: solids, EA: aeration space, AD: available water, AR: remaining water, DU: humid bulk density, DS: dry bulk density, DP: particles distribution. 
Mudas de gérbera (Gerbera jamesonii L.) com quatro folhas definitivas, cultivares Red e Cherry da empresa Sakata Seed Sudamérica $\left(\right.$ Sakata $\left.^{\circledR}\right)$, foram transplantadas em vasos com capacidade para $1 \mathrm{~L}$, preenchidos com os substratos, de acordo com a densidade úmida.

Após o transplante, as mudas foram aclimatadas durante 30 dias, dispostas sobre bancada de madeira, sob malha termorefletora (Aluminet ${ }^{\circledR}$ ), com 50\% de sombreamento, disposta na parte superior interna da casa de vegetação, que mantinha a intensidade luminosa próxima a $25.000 \mathrm{~lx}$. Encerrado esse período, os vasos foram espaçados de 0,25 em $0,25 \mathrm{~m}$ e distribuídos em duas bancadas, permanecendo na intensidade luminosa máxima de 50.000 lx, controlada por meio da abertura e fechamento da malha termorrefletora. A avaliação experimental foi iniciada após a aclimatação e os resultados são apresentados em dias após aclimatação (DAA).

As plantas foram fertirrigadas diariamente de forma manual, sendo a solução aplicada na porção superior do vaso. O manejo da solução nutritiva baseou-se na pesagem diária dos vasos, os quais foram mantidos na faixa de 50 a $25 \%$ da água disponível no substrato. A quantidade máxima de água disponível no substrato foi definida com base na diferença entre as massas obtidas na capacidade máxima de retenção e a massa seca do substrato. Para obter a capacidade máxima de retenção, um vaso por parcela foi saturado via subirrigação durante 24 horas e após saturação, permitiu-se a drenagem da água gravitacional por 24 horas, quando então foi pesado. Para eliminar a interferência do crescimento da planta, quinzenalmente um vaso por parcela era saturado a fim de obter uma nova capacidade máxima de retenção. A irrigação somente era efetuada quando a massa do vaso correspondia a $25 \%$ da água disponível, elevando-se novamente a $50 \%$.

A solução nutritiva, utilizada por produtor especializado e adaptada por Ludwig (2010), apresentou a seguinte

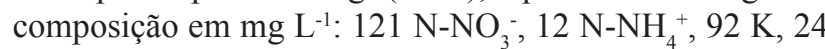
P, $175 \mathrm{Ca}, 27 \mathrm{Mg}$ e $39 \mathrm{~S}, 0,19 \mathrm{~B}, 0,08 \mathrm{Cu}, 2,74 \mathrm{Fe}, 0,19 \mathrm{Mn}$,
0,04 Mo e 0,08 Zn no período vegetativo e $168 \mathrm{~N}-\mathrm{NO}_{3}^{-}, 41$ $\mathrm{N}_{-} \mathrm{NH}_{4}^{+}, 303 \mathrm{~K}, 35 \mathrm{P}, 105 \mathrm{Ca}, 45 \mathrm{Mg}$ e $55 \mathrm{~S}, 0,19 \mathrm{~B}, 0,08$ $\mathrm{Cu}, 2,74 \mathrm{Fe}, 0,19 \mathrm{Mn}, 0,04 \mathrm{Mo}$ e $0,08 \mathrm{Zn}$ no reprodutivo.

A determinação da $\mathrm{CE}$ e do $\mathrm{pH}$ da solução do substrato foi realizada aos 1, 15, 29, 43 e 50 DAA (ponto de comercialização), com o uso das metodologias do "pourthru" de acordo com Cavins et al. (2000), diluição de 1:2 (volume de substrato/volume de água) de acordo com Raij et al. (2001) e diluição de 1:5 (massa de substrato/ volume de água), de acordo com Brasil (2007).

O método "pourthru" consistiu em saturar o substrato com a solução nutritiva aplicada na porção superior do vaso até o início da sua percolação. Estes vasos eram deixados em repouso pelo período de uma hora, quando então recebiam um coletor na sua parte inferior, e a adição de $75 \mathrm{~mL}$ de água destilada na sua porção superior. A água destilada deslocava a solução da parte inferior do vaso, que era coletada e então analisada. Para os métodos de diluição, o substrato foi retirado dos vasos, separado das raízes, homogeneizado e seco ao ar, anteriormente à análise.

Após a coleta da solução pelos métodos, foram determinados os valores de $\mathrm{CE}$ e pH. Para a determinação da condutividade elétrica, foi utilizado condutivímetro portátil da marca Digimed, modelo DM-3; e para o $\mathrm{pH}$, pHmetro portátil da marca Gehaka, modelo PG 1400.

As avaliações foram realizadas aos 1, 15, 29, 43 e 50 DAA, em 40 vasos por dia e 200 vasos no total. As correlações lineares estabelecidas entre as metodologias foram obtidas pelo método de Pearson.

\section{RESULTADOS E DISCUSSÃO}

Os valores de condutividade elétrica (CE) obtidos pelos métodos "pourthru" e as diluições de 1:2 e 1:5 correlacionaram-se positiva e significativamente entre si em todas as datas aferidas (Tabela 2).

Tabela 2. Correlação linear de Pearson entre as metodologias de determinação da condutividade elétrica (CE) do substrato. Botucatu (SP).

Table 2. Linear correlations Pearson's method established among the methodologies for determining electrical conductivity (EC) value of the substrate. Botucatu (SP).

\begin{tabular}{|c|c|c|c|c|c|c|}
\hline & \multicolumn{6}{|c|}{$\mathbf{C E}$} \\
\hline & \multicolumn{2}{|c|}{1 DAA } & \multicolumn{2}{|c|}{15 DAA } & \multicolumn{2}{|c|}{29 DAA } \\
\hline & $\mathrm{CE}_{\mathrm{PT}}$ & $\mathrm{CE}_{1: 5}$ & $\mathrm{CE}_{\mathrm{PT}}$ & $\mathrm{CE}_{1: 5}$ & $\mathrm{CE}_{\mathrm{PT}}$ & $\mathrm{CE}_{1: 5}$ \\
\hline $\mathrm{CE}_{1: 2}$ & $0,639 * *$ & $0,885^{* *}$ & $0,653 * *$ & $0,804 * *$ & $0,453 * *$ & $0,556^{* *}$ \\
\hline \multirow[t]{3}{*}{$\mathrm{CE}_{1: 5}$} & $0,468 * *$ & & $0,735 * *$ & & $0,411 * *$ & \\
\hline & \multicolumn{2}{|c|}{43 DAA } & \multicolumn{2}{|c|}{50 DAA } & & \\
\hline & $\mathrm{CE}_{\mathrm{PT}}$ & $\mathrm{CE}_{1: 5}$ & $\mathrm{CE}_{\mathrm{PT}}$ & $\mathrm{CE}_{1: 5}$ & & \\
\hline $\mathrm{CE}_{1: 2}$ & $0,487 * *$ & $0,727 * *$ & $0,314^{*}$ & $0,720 * *$ & & \\
\hline $\mathrm{CE}_{1: 5}$ & $0,558 * *$ & & $0,394 *$ & & & \\
\hline
\end{tabular}

** significativo a 1\%. * significativo a 5\%. DAA: dias após aclimatação. Metodologias de avaliação: PT: 'pourthru': 1:2: diluição da amostra em duas partes de água; 1:5: diluição da amostra em cinco partes de água.

** significant at 1\%, * significant at 5\%.DAA: days after acclimation. Methodologies: PT: “pourthru”, 1:2 (substrate volume/water volume); 1:5 (substrate mass /water volume). 
Apesar de significativa, a correlação entre o "pourthru" e os métodos de diluição foram inferiores em relação à obtida entre os dois métodos de diluição. Provavelmente, o efeito deva-se à diferença no ponto de amostragem, pois acordo com Sonneveld (2000) os gradientes espaciais dos íons no ambiente radicular podem alterar os resultados. Devido à natureza de deslocamento do método "pourthru", as amostras são obtidas da porção inferior do vaso (WRIGHT,
1986), enquanto para os métodos de diluição, as amostras são obtidas após homogeneização completa do substrato.

Dentre os métodos testados, o "pourthru" apresentou a maior capacidade extrativa, em função dos valores de CE serem superiores aos demais (Figura 2A, 2B e 2C) e mais próximos aos fornecidos pela solução nutritiva. $\mathrm{O}$ fator de diluição afetou proporcionalmente os resultados, concordando com Abreu et al. (2007).

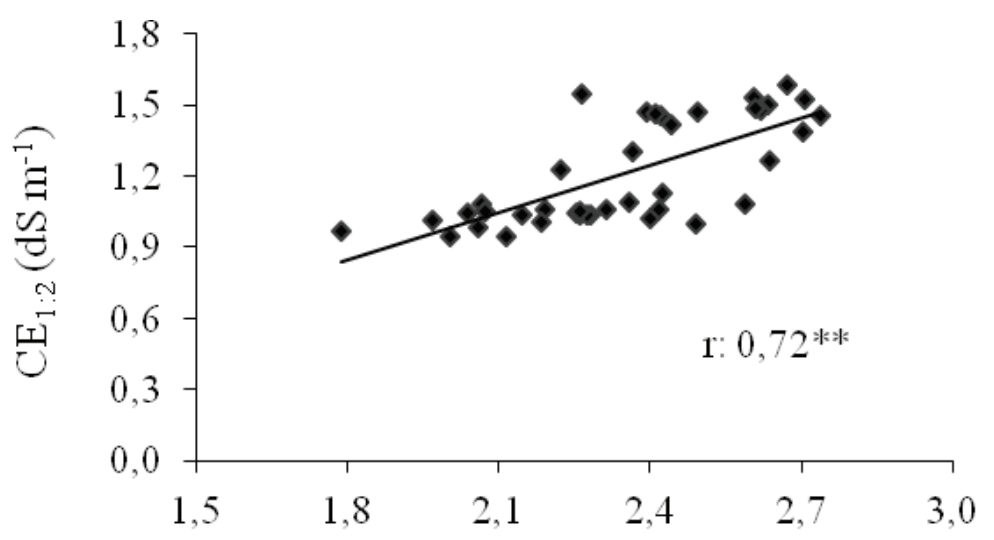

A $\mathrm{CE}_{\text {pourthru }}\left(\mathrm{dS} \mathrm{m}^{-1}\right)$

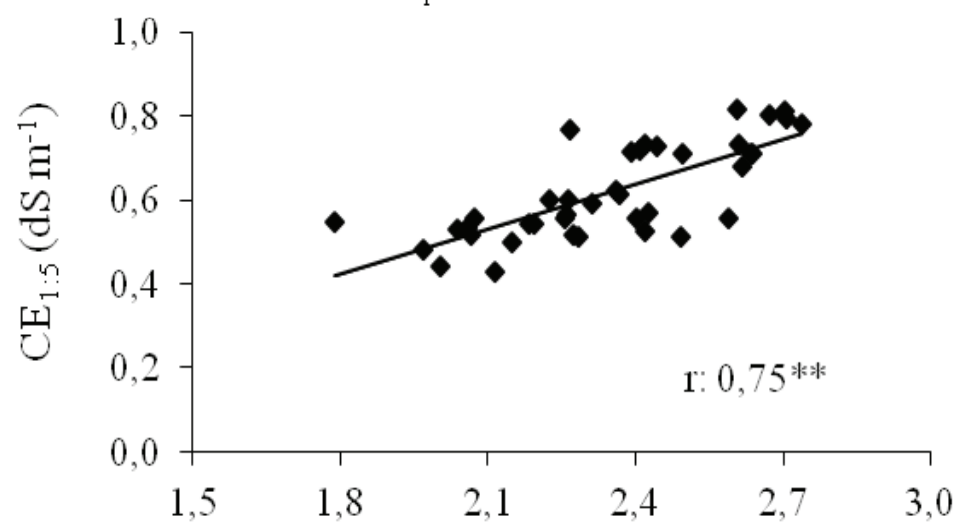

B

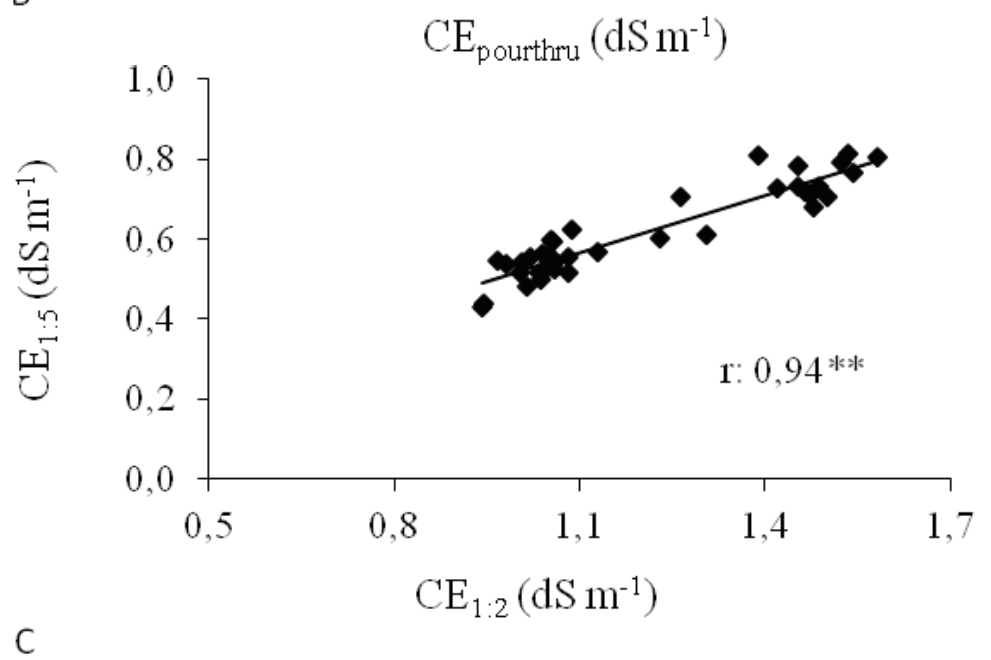

Figura 2. Correlação linear média para a CE da solução do substrato avaliadas pelos métodos do "pourthu" e diluições de 1:2 (A) e 1:5 (B), e diluições do 1:2 e 1:5 (C). Botucatu (SP).

Figure 2. Linear correlation for CE of the substrate solution determined by the methodologies "pourthru”, dilution of 1:2 (A) and 1:5 (B), and dilution of 1:2 and 1:5 (C). Botucatu (SP). 
O "pourthru" não sofre efeito de diluição, pois ocorre somente um arraste da solução disponível no substrato e os resultados podem ser dependentes do sistema de irrigação. Quando a irrigação é realizada manualmente na porção superior do substrato, a água adicionada pode promover o movimento dos nutrientes por fluxo de massa para a parte inferior do vaso (CAVINS et al., 2008) e sendo essa a porção de maior concentração de sais, os valores de CE tendem a se elevar em comparação a métodos com outros pontos de coleta (MOTA et al., 2011).

Aliado à irrigação, as partículas que compõem o substrato também podem interferir nos resultados. Durante o período de cultivo, substratos com partículas bem distribuídas em muitas classes de tamanhos, apresentam o movimento daquelas menores nos espaços formados entre as maiores, acomodando-se e reduzindo a porosidade total (FERNANDES e CORÁ, 2004). Esse ajuste é afetado pelo eventual transporte dos vasos e acentuado pelo deslocamento com a água de irrigação, conferindo maior microporosidade, responsável pela retenção de água e nutrientes, ao substrato localizado na porção inferior do vaso.

Correlação linear positiva e altamente significativa foi verificada entre os métodos para os valores de $\mathrm{pH}$ (Tabela 3, Figura 3A, 3B e 3C). Resultados semelhantes foram obtidos por Ludwig et al. (2007) ao compararem as metodologias do "pourthru" e diluição de 1:2 e por Cavins et al. (2008) com as metodologias do "pourthru" e extrato de saturação. Esses resultados indicam que os valores de pH são pouco influenciados pelo método utilizado, não sendo afetados pela diluição da solução do substrato. Desse modo, sugere-se a utilização do método "pourthru" para determinação dos valores de $\mathrm{pH}$, por ser um método que não exige remoção do substrato, garantindo que não haja distúrbio para o sistema radicular e comprometimento da planta.

Tabela 3. Correlação linear de Pearson entre as metodologias de determinação do valor de pH do substrato. Botucatu (SP).

Table 3. Linear correlations Pearson's method established among the methodologies for determining pH values of the substrate. Botucatu (SP).

\begin{tabular}{|c|c|c|c|c|c|c|}
\hline & \multicolumn{6}{|c|}{ pH } \\
\hline & \multicolumn{2}{|c|}{1 DAA } & \multicolumn{2}{|c|}{15 DAA } & \multicolumn{2}{|c|}{29 DAA } \\
\hline & $\mathrm{pH}_{\mathrm{PT}}$ & $\mathrm{pH}_{1: 5}$ & $\mathrm{pH}_{\mathrm{PT}}$ & $\mathrm{pH}_{1: 5}$ & $\mathrm{pH}_{\mathrm{PT}}$ & $\mathrm{pH}_{1: 5}$ \\
\hline $\mathrm{pH}_{1: 2}$ & $0,962 * *$ & $0,983 * *$ & $0,957 * *$ & $0,988 * *$ & $0,970 * *$ & $0,984 * *$ \\
\hline \multirow[t]{3}{*}{$\mathrm{pH}_{1: 5}$} & $0,939 * *$ & & $0,956 * *$ & & $0,962 * *$ & \\
\hline & \multicolumn{2}{|c|}{43 DAA } & \multicolumn{2}{|c|}{50 DAA } & & \\
\hline & $\mathrm{pH}_{\mathrm{PT}}$ & $\mathrm{pH}_{1: 5}$ & $\mathrm{pH}_{\mathrm{PT}}$ & $\mathrm{pH}_{1: 5}$ & & \\
\hline $\mathrm{pH}_{1: 2}$ & $0,910 * *$ & $0,992 * *$ & $0,973 * *$ & $0,990 * *$ & & \\
\hline $\mathrm{pH}_{1: 5}$ & \multicolumn{2}{|l|}{$0,896 * *$} & \multicolumn{2}{|l|}{$0,975 * *$} & & \\
\hline
\end{tabular}

Os valores de $\mathrm{pH}$ das soluções dos substratos variaram entre 5,0 e 7,0 (Figura 3), com pouca alteração média ao longo do ciclo. Esta variação deve-se à diferença na aplicação de corretivos de acidez pelas empresas fornecedoras dos substratos, conforme verificado na caracterização inicial (Tabela 1).

A elevada porosidade, a boa drenagem e a baixa densidade permitem ao "pourthru" ser considerado um método ideal para deslocamento da solução do substrato em recipientes (CAVINS, 2002). De acordo com Mota et al. (2011), em substratos com menor espaço de aeração e maior retenção de água, a quantidade de agua para promover o deslocamento e o tempo de estabilização podem sofrer alteração. Porém, a reprodutibilidade dos resultados obtida, indica que as características físicas destes substratos não limitaram a utilização dos métodos.

Entretanto, a fim de validar o método "pourthru" e permitir sua utilização em substratos com diferentes características físicas de espaço de aeração e retenção de água, é necessário que novos trabalhos sejam desenvolvidos, considerando uma ampla faixa de porosidade dos materiais disponíveis no mercado. 


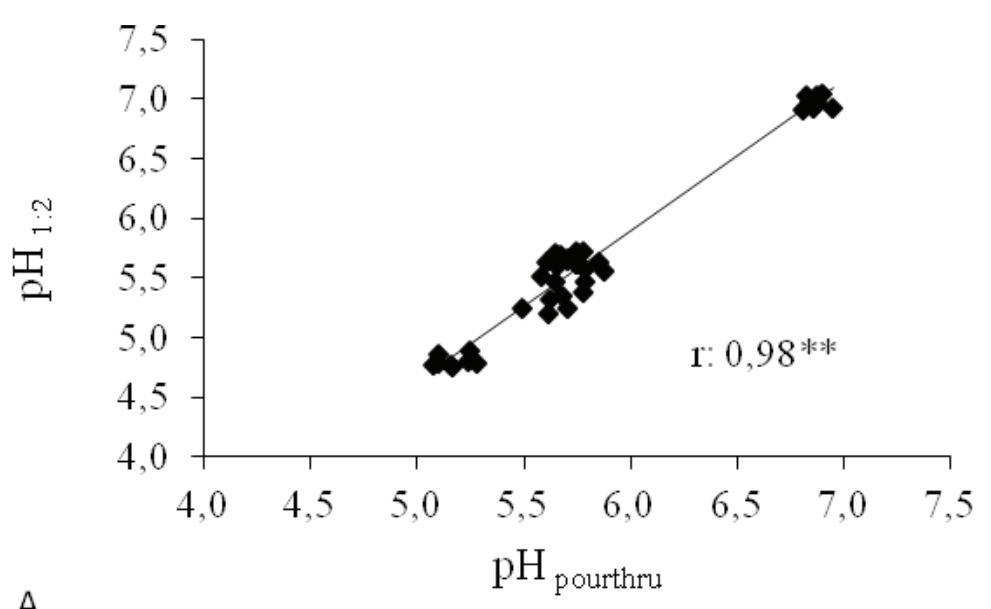

A
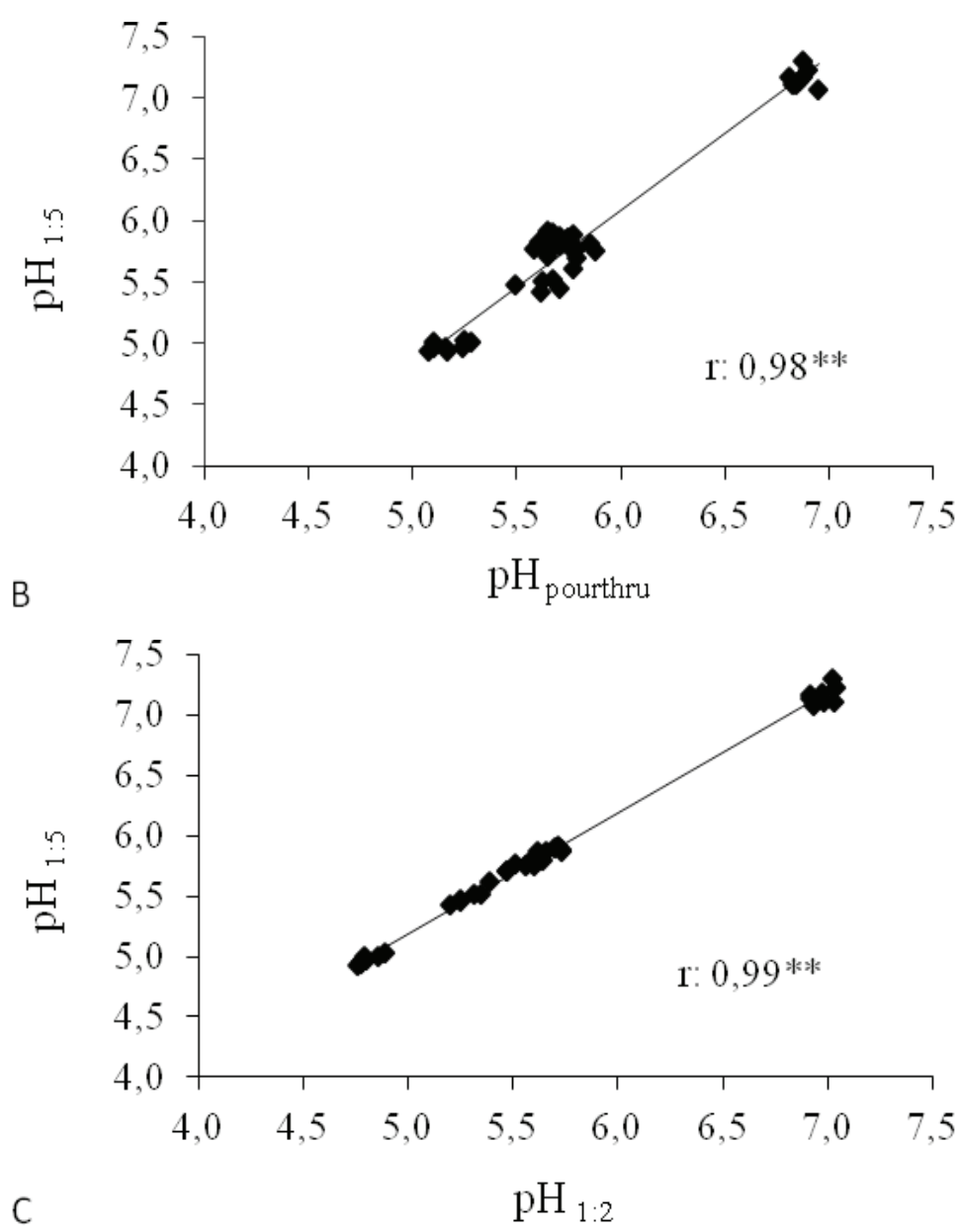

Figura 3. Correlação linear média o pH da solução do substrato avaliadas pelos métodos do "pourthu" e diluições de 1:2 (A) e 1:5 (B), e diluições do 1:2 e 1:5 (C). Botucatu (SP).

Figure 3. Linear correlation for $\mathrm{pH}$ of the substrate solution determined by the methodologies "pourthru", dilution of 1:2 (A) and 1:5 (B), and dilution of 1:2 and 1:5 (C). Botucatu (SP). 


\section{CONCLUSÕES}

As correlações significativas para os valores de $\mathrm{CE}$ e $\mathrm{pH}$ validam os métodos para avaliação nutricional em plantas de gérbera conduzidas em substrato.

O método "pourthru" pode ser indicado para o monitoramento nutricional periódico da solução do substrato para a cultura da gérbera, por ser não destrutivo e agilizar a obtenção da resposta.

\section{REFERÊNCIAS}

ABREU, M.F.;ABREU, C.A.; SARZI, I.; PADUAJUNIOR, A.L. Extratores aquosos para a caracterização química de substratos para plantas. Horticultura Brasileira, Brasília, v.25, n.2, p.184-187, 2007.

BRASIL. Instrução Normativa n. 17, de 21 de maio de 2007. Aprova os Métodos Analíticos Oficiais para Análise de Substratos e Condicionadores de Solos. Diário Oficial da União, Brasília, 24 maio. 2007. Seção 1, p.8.

CAVINS, T.J.; WHIPKER, B.E.; FONTENO, W.C.; HARDEN, B.; McCALL, I.; GIBSON, J.L. Monitoring and managing $\mathrm{pH}$ and EC using the PourThru extraction method. Raleigh: Horticulture Information, v.590, p.1-17, 2000.

CAVINS, T.J. WHIPKER, B.E.; FONTENO, W.C. Establishment of calibration curves for comparing pour-through and satured media extract nutrient values. HortScience, Alexandria, v.39, p.1635-1639, 2004.

CAVINS, T.J. Adaptation of the pourthru nutrient extraction procedures to greenhouse crop production. 2002. 148f. Tese (Doutorado) - Faculty of North Carolina State University, Raleigh.

CAVINS, T.J.; WHIPKER, B.E.; FONTENO, W.C. Timing of PourThru affects $\mathrm{pH}$, electrical conductivity, and leachate volume. Communications in Soil Science and Plant Analysis. New York, v.36, n.11/12, p.1573-1581, 2005.

CAVINS, T.J.; WHIPKER, B.E.; FONTENO, W.C. PourThru: A method for monitoring nutrition in the greenhouse. Acta Horticulturae, Wageningen, v.779, p.289-298, 2008.

DE BOODT, M.; VERDONCK, O. The physical properties of the substrates in horticulture. Acta Horticulturae, Wageningen, v.26, n.4, p.37-44, 1972.
FERNANDES, C.; CORÁ, J.E. Bulk density and relationship air/water of horticultural substrate. Scientia Agricola, Piracicaba, v.61, n.4, p.446-450, 2004.

LUDWIG，F.; FERNANDES，D.M.; MOTA， P.R.D.; VILLAS BÔAS, R.L. Condutividade elétrica e $\mathrm{pH}$ do substrato em cultivares de gérbera de vaso avaliado com duas metodologias. In: CONGRESSO BRASILEIRO DE FLORICULTURA E PLANTAS ORNAMENTAIS, 16, Goiânia, 2007, Anais... Goiânia, 2007.

LUDWIG, F. Características dos substratos no desenvolvimento, nutrição e produção de gérbera (Gerbera jamesonii) em vaso. Botucatu: Faculdade de Ciências Agronômicas, UNESP, 2010. 79p. Tese (Doutorado em Agronomia/Horticultura).

MERCURIO, G. Gerbera cultivation in greenhouse. The Netherlands: Schreurs, 2002. 206p.

MOTA, P.R.D.; VILLAS BÔAS, R.L.; LUDWIG, F.; FERNANDES, D.M.; FOLEGATTI, M.V. Avaliação do $\mathrm{pH}$ e da condutividade elétrica do substrato sob níveis de fertirrigação e métodos de extração da solução. Revista Brasileira de Horticultura Ornamental, Campinas, v.17, n.2, p.121-126, 2011.

RAIJ, B. van; ANDRADE, J.C.; CANTARELLA, H.; QUAGGIO, J. A. Análise química para avaliação da fertilidade de solos tropicais. Campinas: Instituto Agronômico, 2001. 284p.

SANTOS, P.H. Avaliação de métodos de extração de micronutrientes em substratos para as plantas. 2005. 67f. Dissertação (Mestrado em Agricultura Tropical e Subtropical) - Instituto Agronômico de Campinas, Campinas, SP.

SONNEVELD, C.; ELDEREN, C.W. Chemical analysis of peaty growing media by means of water extraction. Communication on Soil Science and Plant Analysis, New York, v.25, n.19/20, p.3199-3208, 1994.

SONNEVELD, C. Effects of salinity on substrate grown vegetables and ornamentals in greenhouse horticulture. 2000. 151f. Tese (Doutorado) - Wageningen Universitiet, Netherlands.

WRIGHT, R.D. The Pour-through nutrient extraction procedure. HortScience, Alexandria, v.21, n.2, p.227-229, 1986. 